\title{
Anticoncepción y aborto en Argentina: perspectivas de obstetras y ginecólogos
}

\author{
Dalia Szulik, Soc, (') Mónica Gogna, Soc, (2) Mónica Petracci, Soc, ${ }^{(3)}$ \\ Silvina Ramos, Soc, (4) Mariana Romero, Soc. ${ }^{(2)}$
}

\section{Szulik D, Gogna M, Petracci M, Ramos S, Romero M. Anticoncepción y aborto en Argentina: perspectivas de obstetras y ginecólogos. \\ Salud Publica Mex 2008;50:32-39.}

\section{Resumen}

Objetivo. Reflexionar sobre el rol de los tocoginecólogos/as en torno a las políticas públicas en salud reproductiva en Argentina. Material y métodos. Combinación de métodos cuantitativos (encuesta, $n=467$ ) y cualitativos (entrevista semiestructurada, $n=35$; grupos focales, $n=6$ ). Resultados. Para los profesionales, el aborto y la anticoncepción son problemas muy relevantes. Siete de 10 otorgaron máxima prioridad a implementar servicios de planificación familiar y consejería anticonceptiva pos-aborto. Uno de 2 propuso promover el debate social sobre aborto. La gran mayoría acordó que despenalizar el aborto contribuiría a disminuir la mortalidad materna y que la ley no debería penalizarlo ante riesgo de vida o salud, violación o incesto y malformación incompatible con la vida extrauterina. Conclusiones. Las cuestiones más críticas del campo de la salud reproductiva forman parte de la agenda de los médicos. Las acciones de abogacía deben profundizar una visión integral de la salud y destacar la responsabilidad social de estos actores.

Palabras claves: tocoginecólogos; políticas públicas; anticoncepción; aborto; responsabilidad profesional; Argentina
Szulik D, Gogna M, Petracci M, Ramos S, Romero M.

Contraception and abortion in Argentina:

Perspective of obstetricians and gynaecologists.

Salud Publica Mex 2008;50:32-39.

\section{Abstract}

Objective. To explore the role of obstetricians and gynaecologists in reproductive public health policies in Argentina. Material and Methods. Combination of quantitative methods (survey, $n=467$ ) and qualitative methods (semistructured interview, $n=35$; focus groups, $n=6$ ). Results. The great majority of respondents believe that abortion and contraception are serious public health issues. Seven out of 10 considered the implementation of family planning services and post-abortion contraceptive counseling to be priorities. One-half favored promoting social debate on abortion. The great majority thought that de-criminalization of abortion would help to diminish maternal mortality and that abortion should not be penalized when the woman's life or health is at risk, or in cases of rape or fetal malformations. Conclusions. Abortion and contraception are important issues for physicians. Advocacy efforts within this community need to focus on an integral vision of health, emphasizing their social responsibility.

Key words: obstetricians; gynaecologists; public policies; contraception; abortion; professional responsibility;Argentina

La investigación que dio origen a este artículo fue financiada por el Programa de Reproducción Humana de la Organización Mundial de la Salud y la Secretaría de Ciencia y Técnica del Ministerio de Educación y Cultura de la Nación. Por otra parte, fue auspiciada por la Secretaría de Salud del Gobierno de la Ciudad de Buenos Aires y la Sociedad de Ginecología y Obstetricia de Buenos Aires.

(I) Facultad Latinoamericana de Ciencias Sociales, Argentina; Consejo Nacional de Investigaciones Científicas y Tecnológicas, Argentina.

(2) Centro de Estudios de Estado y Sociedad; Consejo Nacional de Investigaciones Científicas y Tecnológicas, Argentina.

(3) Centro de Estudios de Estado y Sociedad; Instituto de Investigaciones Gino Germani, Universidad de Buenos Aires, Argentina.

(4) Centro de Estado y Sociedad, Argentina.

Fecha de recibido: 5 de enero de 2006 • Fecha de aprobado: 4 de septiembre de 2006

Solicitud de sobretiros: Mónica Gogna. Centro de Estudios y Sociedad. Sánchez de Bustamante 27 (CI I 73AAA). Buenos Aires, Argentina. Correo electrónico: salud@cedes.org 
$\mathrm{E}$ ste artículo se centra en el rol que juegan los tocoginecólogos/as en el debate social sobre políticas públicas en el campo de la salud reproductiva en Argentina. El peso de la comunidad médica en la escena pública ha sido destacado en diferentes momentos históricos y contextos. Por ejemplo, en 1850 en Estados Unidos, los médicos lideraron la cruzada antiaborto. Conrad y Schneider ${ }^{1}$ describen cómo, con el propósito de ayudar a su naciente profesionalización y crear un monopolio para su accionar, estos profesionales hicieron un eficaz lobby para convencer a los legisladores del peligro del aborto y su inmoralidad. Como subproducto de la penalización del aborto en varios estados, la opinión pública, antes indiferente o tolerante, endureció sus actitudes. Otro ejemplo en el siglo XX fue el rol de los médicos en las "cruzadas" higienistas y de control de la natalidad.

Frecuentemente, los médicos vieron sus valores como aquéllos que debían guiar el comportamiento de los otros, argumentando, en nombre de la ciencia, ciertas posiciones de índole moral. También los ginecólogos y obstetras han desempeñado un papel fundamental en la creación de leyes o normativas para mejorar la calidad de la atención obstétrica y en los procesos de liberalización de leyes y reglamentos sobre el aborto en muchos países. ${ }^{2-4}$ En la Argentina, su presencia en la esfera pública ha tenido ribetes difusos y un bajo perfil.

El objetivo de este artículo es presentar, a partir de los hallazgos de una investigación acerca de sus perspectivas sobre anticoncepción y aborto, ${ }^{5}$ algunas ideas que permitan comprender el "caso argentino" y delinear líneas para el trabajo de abogacía con estos profesionales.

La problemática de la salud y los derechos sexuales y reproductivos en la Argentina ha atravesado en las últimas décadas un proceso de transición: desde una situación de restricciones y omisiones hacia una de mayor visibilidad pública, con paulatinos cambios institucionales y programáticos en los diversos niveles de la política pública. El recorrido ha sido desigual en lo que atañe a la anticoncepción y al aborto. ${ }^{6}$ En el primer caso, se ha pasado de una situación en la cual las actividades destinadas al control de la natalidad estaban prohibidas (década de los setenta) y los programas públicos de salud estaban permeados por una clara ideología pronatalista a un escenario en el que el Estado se ha comprometido a garantizar a la población el acceso a la anticoncepción.

Durante los años noventa, con fuerte influencia de los consensos de Cairo y Beijing, la salud reproductiva ingresó en la agenda pública debido en gran medida al accionar del movimiento de mujeres. En la mayoría de las provincias se aprobaron leyes que impulsan la creación de programas de salud reproductiva y reconocen el derecho de las personas a regular la fecundidad. Finalmente, luego de un largo proceso, en 2002 se creó el Programa Nacional de Salud Sexual y Procreación Responsable. Anteriormente había sido imposible debido a la presión de la iglesia católica y la posición refractaria del gobierno del ex presidente Menem. ${ }^{7}$

La comunidad médica no fue ajena a estos acontecimientos, pero su participación se restringió a la intervención de algunos médico/as prestigiosos/as en los medios de comunicación y en instancias de consulta por parte de los legisladores. En 2003 las principales sociedades científicas y profesionales publicaron en diversos medios gráficos nacionales una solicitada en apoyo al Programa Nacional, a propósito de las acciones legales iniciadas por los sectores más conservadores de la iglesia católica, que solicitaban la suspensión de los programas de salud reproductiva, la derogación de las leyes y cuestionaban la entrega de métodos anticonceptivos a adolescentes y la provisión a la anticoncepción de emergencia. La solicitada destacaba la necesidad de que la política pública diera una respuesta seria a las altas cifras de morbimortalidad materna, las tasas crecientes de mortalidad por cáncer génitomamario y el aumento de los egresos hospitalarios por complicaciones de aborto. ${ }^{8}$

Con respecto al aborto, durante el período analizado no se produjeron innovaciones legislativas ni programáticas. Al igual que en la mayor parte de los países de América Latina, en la Argentina el aborto es objeto de una legislación restrictiva. El Código Penal Argentino tipifica al aborto como un delito contra la vida y la persona. Establece penas de reclusión o prisión para el profesional que lo efectúa y para la mujer que se causara o consintiera un aborto. Sólo hay dos causales de despenalización: si el aborto se ha hecho con el fin de evitar un peligro para la vida o la salud de la madre y si este peligro no puede ser evitado por otros medios; o si el embarazo proviene de una violación o de un atentado al pudor cometido sobre una mujer idiota o demente. Sin embargo, es inusual que las mujeres accedan a interrumpir un embarazo en el sector público. Ello hace que prácticamente funcione una prohibición absoluta del aborto. ${ }^{9}$

El tema del aborto está instalado en la agenda de debate desde 1994 cuando el entonces presidente Menem intentó introducir en el texto de la Constitución una cláusula sobre el derecho a la vida desde el momento de la concepción. Tanto el movimiento de mujeres como algunos sectores estatales y gubernamentales, los medios de comunicación y la opinión pública cuestionaron la 
maniobra, pero legitimaron la necesidad de discutir el tema, básicamente debido a sus implicancias en términos de salud pública y de derechos. ${ }^{10}$

En este debate, la única manifestación de una entidad representativa del campo médico fue una declaración de la Academia Nacional de Medicina en la que se sostenía que "la vida humana comienza con la fecundación". Rechazaba la idea de que la legalización del aborto disminuyera la morbimortalidad materna o que terminara con su clandestinidad. ${ }^{11} \mathrm{Ni}$ las facultades de medicina ni las sociedades científicas emitieron opinión institucional alguna.

\section{Material y métodos}

Los sujetos de la investigación fueron médicos/as que se desempeñan en los servicios de ginecología, obstetricia y tocoginecología de los 15 establecimientos asistenciales públicos de la ciudad de Buenos Aires y en 14 hospitales generales y materno-infantiles de seis partidos del Gran Buenos Aires. El estudio fue exploratorio-descriptivo con un diseño basado en la complementación de métodos cuantitativos y cualitativos. El trabajo de campo se realizó entre septiembre y diciembre de 1998.

Se emplearon tres técnicas de relevamiento, previa aplicación de consentimientos informados: entrevista semiestructurada, encuesta autoadministrada y grupos focales. La entrevista $(n=35)$ fue aplicada a los jefes/as de los servicios con el objetivo de sondear las orientaciones e indicaciones de los máximos responsables hacia el personal a cargo y obtener información del contexto institucional. Tres jefes/as en la ciudad de Buenos Aires, y tres en el Gran Buenos Aires rechazaron la aplicación de la encuesta en sus servicios.

La encuesta autoadministrada $(n=467$, tasa de respuesta $83 \%{ }^{*}$ ) fue aplicada a todo el personal permanente de los servicios: médicos/as de planta (59\%), residentes (31\%) y médicos/as de guardia (10\%). Su objetivo fue recuperar sus opiniones sobre diversos aspectos de la salud reproductiva. Veinticinco hospitales aceptaron participar del estudio, $67 \%$ de las encuestas se realizaron en establecimientos de la ciudad de Buenos Aires y 33\% en hospitales del Gran Buenos Aires. Sesenta por ciento son mujeres y $40 \%$ hombres; su edad promedio es 39 años. Se realizaron seis grupos focales con médicos de

\footnotetext{
* Se entregó la encuesta a 561 médicos/as, incluyendo las encuestas "perdidas" (85 casos) y los "rechazos" (5 hombres y 4 mujeres que rechazaron participar del estudio a través del consentimiento informado).
}

ambos sexos para iluminar algunas "cajas negras" de los resultados de la fase cuantitativa y para explorar ciertas cuestiones que necesariamente deben ser abordadas con metodología cualitativa. El perfil sociodemográfico de los 46 participantes de los grupos focales fue el siguiente: $54 \%$ mujeres y $46 \%$ varones. En cuanto a la edad, la distribución fue la siguiente: 25-34 años: 63\%; 35-44 años: 20\%; 45 años y más: $17 \%$.

Con respecto al análisis de los datos, para los resultados de la encuesta se utilizaron técnicas descriptivas simples y análisis bivariado. En cuanto a la información cualitativa, los ejes de análisis surgieron de las respectivas pautas-guía, recuperando las dimensiones que emergían como relevantes en el proceso de lectura y relectura de las transcripciones. Se retomaron los consensos y las controversias, intentando explicitar las dimensiones o argumentos sobre las cuales se construyeron acuerdos y disensos.

\section{Resultados}

El nivel de relevancia para la salud pública atribuido a un conjunto de problemas de la salud reproductiva -aborto, abuso sexual, anticoncepción, anticoncepción quirúrgica, cáncer genitomamario, cesárea, controles prenatales, embarazo adolescente, enfermedades de transmisión sexual, infertilidad, nutrición, VIH/SIDA y violencia doméstica- fue indagado a través de una escala de cinco ítems. La relevancia del aborto para la salud pública fue consensuada por los profesionales entrevistados (cuadro I). El 65\% le asignó el máximo grado de relevancia, porcentaje que alcanza $78 \%$ al tomar conjuntamente las categorías muy y bastante relevante. Otros dos problemas considerados muy relevantes fueron la anticoncepción (51\%) y el cáncer genitomamario (50\%).

Con el propósito de conocer las opiniones de los médicos/as acerca de las orientaciones de las políticas en el campo estudiado, se indagó acerca del grado de prioridad adjudicado a un conjunto de acciones (cuadro II). Las acciones consideradas por la amplia mayoría como muy prioritarias son la educación sexual, la prevención de VIH/SIDA y la regulación de la fecundidad. Estas problemáticas fueron definidas por más de $70 \%$ de los médicos/as como "prerrogativas de todo individuo que el Estado debe asegurar a través de leyes, programas y servicios".

La implementación de programas de educación sexual dirigidos a la población adolescente es, para 9 de cada 10 respondentes, un deber ser de la política pública. Esta opinión se ve reforzada por el acuerdo de casi la totalidad de los encuestados (98\%) con la frase: "la educación sexual contribuye a que los jóvenes tengan 
Cuadro I

Nivel de relevancia atribuido a problemas de la salud pública en el país ( $N=467$ ). Buenos Aires, SEPTIEMBRE-DICIEMBRE DE 1998

\begin{tabular}{|c|c|c|c|c|c|c|}
\hline & $\begin{array}{c}\text { Muy relevante } \\
\%\end{array}$ & $\begin{array}{c}\text { Bastante relevante } \\
\%\end{array}$ & $\begin{array}{c}\text { Relevante } \\
\%\end{array}$ & $\begin{array}{c}\text { Poco relevante } \\
\%\end{array}$ & $\begin{array}{c}\text { Nada relevante } \\
\%\end{array}$ & $\begin{array}{c}\text { No sabe/ no contesta } \\
\%\end{array}$ \\
\hline Aborto & 65 & 13 & 12 & 7 & 2 & I \\
\hline Anticoncepción & 51 & 14 & 18 & 10 & 4 & 3 \\
\hline Cáncer genitomamario & 50 & 20 & 22 & 4 & 1 & 3 \\
\hline $\mathrm{VIH} /$ sida & 48 & 22 & 20 & 8 & I & 1 \\
\hline Controles prenatales & 48 & 18 & 20 & 8 & 2 & 4 \\
\hline Embarazo adolescente & 43 & 27 & 16 & 10 & 2 & 2 \\
\hline ETS & 40 & 25 & 21 & 10 & 2 & 2 \\
\hline Abuso sexual & 34 & 22 & 25 & 13 & 3 & 3 \\
\hline Nutrición & 33 & 19 & 24 & 15 & 7 & 2 \\
\hline Violencia doméstica & 25 & 20 & 26 & 17 & 8 & 4 \\
\hline Cesárea & 16 & 21 & 39 & 17 & 3 & 4 \\
\hline Anticoncepción quirúrgica & 6 & 7 & 19 & 36 & 25 & 7 \\
\hline Infertilidad & 5 & 14 & 37 & 30 & 10 & 4 \\
\hline
\end{tabular}

Cuadro II

Nivel de prioridad asignado a las acciones en salud pública ( $N=467)$. Buenos Aires, SEPTIEMBRE-DICIEMBRE DE 1998

\begin{tabular}{|c|c|c|c|c|}
\hline & $\begin{array}{c}\text { Muy prioritario } \\
\%\end{array}$ & $\begin{array}{c}\text { Prioritario } \\
\%\end{array}$ & $\begin{array}{c}\text { Poco/nada prioritario } \\
\%\end{array}$ & No sabe Ino contesta \\
\hline Implementar programas de educación sexual dirigidos a la población adolescente & 87 & II & I & I \\
\hline Implementar campañas de prevención de $\mathrm{VIH} /$ sida con distribución de preservativos & 82 & 12 & 5 & I \\
\hline Implementar y/o mejorar los programas de asistencia en anticoncepción & 73 & 25 & I & I \\
\hline Implementar programas de asesoramiento anticonceptivo postaborto & 71 & 24 & 4 & I \\
\hline Promover el debate público sobre la situación del aborto en el país & 48 & 34 & 16 & 2 \\
\hline Implementar programas para mejorar la atención de los abortos hospitalizados & 34 & 40 & 23 & 3 \\
\hline
\end{tabular}

una vida sexual más responsable y segura". En cuanto a la anticoncepción, la implementación de servicios de planificación familiar y la consejería anticonceptiva pos-aborto resultan muy prioritarias para 7 de cada 10 profesionales. Es posible relacionar este dato con la opinión vertida acerca de la atención que reciben las mujeres internadas por complicaciones de aborto: $60 \%$ manifestó que "a veces" son asesoradas sobre anticoncepción antes del alta mientras que sólo 2 de cada 10 afirmaron que esto ocurre "siempre" (cuadro III).

De forma similar, el nivel de prioridad asignado a la implementación de programas para mejorar la atención de los abortos hospitalizados es consistente con la eva- luación general que los profesionales hacen de la atención de las complicaciones de aborto en los hospitales públicos. El 56\% opinó que esta atención es muy buena o excelente. Dicha calificación se refiere básicamente a la eficiencia y la calidad médica con que se resuelve el motivo de internación, no al reconocimiento de que se ofrece una atención integral, que incluiría que la mujer sea tratada como un sujeto con derechos y contenida desde el punto de vista psicológico (cuadro III).

Para aproximarnos a la visión de los profesionales sobre la dimensión pública y política del aborto, recurrimos a un conjunto de preguntas y frases acerca de las cuales los encuestados debían expresar su acuerdo 
Cuadro III

Opinión acerca de la atención en el hospital público por COMPLicaciones de aborto ( $\mathrm{N}=467$ ). Buenos Aires, SEPTIEMBRE-DICIEMBRE DE 1998

\begin{tabular}{|c|c|c|c|c|}
\hline & $\begin{array}{c}\text { Siempre } \\
\%\end{array}$ & $\begin{array}{c}A \text { veces } \\
\%\end{array}$ & $\begin{array}{c}\text { Nunca } \\
\%\end{array}$ & $\begin{array}{c}\text { No sabe } \\
\%\end{array}$ \\
\hline Es tratada con eficiencia y calidad médica & 77 & 20 & I & 2 \\
\hline Es tratada como una paciente con derechos & 48 & 41 & 7 & 4 \\
\hline Es asesorada sobre anticoncepción antes del alta & 23 & 60 & 12 & 5 \\
\hline Es contenida psicológicamente & 12 & 63 & 21 & 4 \\
\hline
\end{tabular}

o desacuerdo. Se observa que prácticamente uno de cada dos encuestados consideró muy prioritario promover el debate social acerca de esta problemática (cuadro II).

Veamos ahora qué opinan sobre la penalización de la interrupción del embarazo y eventuales cambios en la legislación vigente. Se les plantearon seis situaciones respecto de las cuales indicaban si la ley debería o no penalizar la interrupción voluntaria del embarazo (cuadro IV). Existe un amplio acuerdo (superior a 80\%) en que la ley no debería penalizar la interrupción de la gestación en caso de riesgo de vida o de salud de la mujer, violación o incesto y en caso de malformación incompatible con la vida extrauterina. Sólo la primera de estas situaciones está contemplada como excepción en el Código Penal. Es decir, que la gran mayoría de los encuestados estaría en principio dispuesto a una ampliación de las actuales excepciones.

El acuerdo con la no penalización disminuye considerablemente en aquellas situaciones en las que no existe riesgo biopsicológico, o éste no es tan evidente. Sólo tres de cada diez médicos/as estuvieron de acuerdo con la no penalización en caso de decisión autónoma de la mujer (38\%) y de condición social y económica adversa $(32 \%)$. En este último caso, el acuerdo es mayor entre las mujeres $(41 \%)$ que entre los varones $(29 \%)(p=.009)$. Finalmente, cabe recalcar que aun cuando la gran mayoría $(85 \%)$ está en desacuerdo con la penalización absoluta del aborto, existe un porcentaje minoritario de profesionales (9\%) que acuerda con dicha frase; que no acepta ni siquiera las excepciones contempladas en la legislación vigente.

Para complementar la descripción de estas posturas, exploramos la opinión de los médicos/as respecto de una estrategia que, desde la perspectiva de salud pública, tendría un efecto positivo sobre la reducción de la mortalidad materna: la despenalización del aborto. Ocho de cada 10 acordaron con que "la despenalización contribuiría a disminuir las muertes maternas". El acuerdo también en este caso es mayor entre las mujeres $(87 \%)$ que entre los varones $(80 \%)(p=.038)$.

En los grupos focales exploramos cómo interpretaban los médicos/as el hecho de que, habiendo tal grado de consenso sobre los efectos positivos de la despenalización del aborto, la comunidad profesional no se hubiera manifestado al respecto cuando el debate sobre el aborto cobró estado público. Este acuerdo no logra plasmarse en un cuestionamiento a la actual legislación restrictiva. Las opiniones están sólo parcialmente influenciadas por el eventual impacto de la despenalización sobre la mortalidad materna (argumento "técnico" o de "salud pública"). En todos los casos, los discursos están fuertemente atravesados por una voz "normativa". ${ }^{12}$

$\mathrm{PM}^{*}$ : La opinión (acuerdo con la frase) es porque estamos todos de acuerdo en eso. Ahora.... ir a poner la cara es ser una kamikaze.

PV: Tenés que agarrar un periodista y decir ¿cuántos números marca para lograr que una persona vaya a un debate público sobre el aborto? Ahí te vas a dar cuenta que es un problema de jerarquía. No poner la cara porque va a traer represalias, por tus pares, por tu hospital, por tu sociedad científica...

PV: Es ser rotulado.

PV: Creo que si uno esboza un razonamiento sobre el aborto y su problemática, que hay que solucionarlo, etc, etc, el primer mote es que es un abortero, aunque esté en desacuerdo... (Servicio de Obstetricia, Hospital I, grupo de médicos de planta)

PV: Creo que si se despenaliza el aborto hay un riesgo que se malentienda un poco la despenalización del aborto, que se haga en forma indiscriminada... (Servicio de Ginecología, Hospital S, grupo de residentes).

* PV: participante varón; PM: participante mujer. 
Cuadro IV

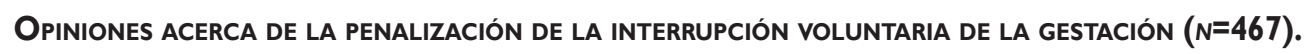
Buenos AIRES, SEPTIEMBRE-DICIEMBRE DE 1998

\begin{tabular}{|c|c|c|c|}
\hline & $\begin{array}{c}\text { Acuerdo } \\
\%\end{array}$ & $\begin{array}{c}\text { Desacuerdo } \\
\%\end{array}$ & $\begin{array}{c}\text { No sabe/ no contesta } \\
\%\end{array}$ \\
\hline La ley debería penalizar la interrupción de la gestación en todos los casos & 9 & 85 & 6 \\
\hline \multicolumn{4}{|l|}{ La ley no debería penalizar la interrupción de la gestación en caso de ... } \\
\hline Riesgo de vida o de salud de la mujer & 87 & 8 & 5 \\
\hline Violación o incesto & 83 & II & 6 \\
\hline Malformación incompatible con la vida extrauterina & 82 & 13 & 5 \\
\hline Decisión autónoma de la mujer & 38 & 52 & 10 \\
\hline Condición social y económica adversa & 32 & 57 & II \\
\hline
\end{tabular}

PV: Y sí, generalmente lo que frenó, lo que vos decís, la Constitución.... para la legalización del aborto....

PM: El poder que tiene la Iglesia en este país.

PV: Obviamente no manifiesto, pero sí, la política...... pero sí, atrás de ellos está la Iglesia haciendo fuerza para que esto no llegue.

PM: Tienen poder político.

PV: Y yo creo que los médicos no lo aceptan más que nada por eso, no porque no estén convencidos de que quizás está bien. (Servicio de Ginecología, Hospital M, grupo de residentes)

Las posturas más permeables a la despenalización del aborto, ejemplificadas aquí por algunos jefes de servicio, suelen combinar el argumento "de salud pública" con el de la "equidad social".

Si le falla el método anticonceptivo...no hay ningún método que sea $100 \%$ seguro. Y si le falla por equis motivo, yo creo que también tendría derecho esa mujer a decidir si lo va a tener o no lo va a tener, y que se atienda como la gente. Porque eso, los que tienen recursos económicos no tienen ningún problema, y el que no tiene recursos cae en eso (tocoginecólogo, Gran Buenos Aires)

Las pacientes del nivel social de los que dictan leyes, de los parlamentarios, pueden acceder a cualquier cosa, incluso a hacerse un aborto provocado en el más lujoso sanatorio de la Capital Federal, así que eso es una hipocresía social (tocoginecólogo, Gran Buenos Aires)

En un grupo de médicos/as de planta, mayoritariamente compuesto por mujeres, la discusión estuvo planteada en términos que podrían considerarse más cercanos a la perspectiva feminista.
PM: (el aborto) es una decisión de la mujer porque es el cuerpo de la mujer.

PM: La que se banca es la mujer y punto.

PM: A mí nadie me va a venir a decidir por mí.... voy a escuchar la opinión de mi pareja, todo eso, pero, te puede influenciar y todo, sí, pero la decisión última es de la mujer.

PV: Quien lo padece.

PM: Además que siempre viene la mujer sola, en general. No viene acompañada de la pareja, en el hospital y en privado, es raro que venga con la pareja. Vienen solas. Entonces la decisión la toma sola. (Servicio de Ginecología, Hospital Q, grupo de médicos de planta)

Aquello que los médicos/as imaginan ser la postura dominante entre "los legos" ejerce una influencia importante sobre lo que están dispuestos a afirmar públicamente.

PM: Uno ve que la gente que está externa a la medicina se empecina mucho más en ponerse en contra del aborto que la gente que lo ve, que lo vive.

PM: Miedo a que si alguien toca un tema tan importante y la sociedad va a estar tan dividida, uno va a perder el poder que ya tiene.

PM: A quedar etiquetado.

PV: Uno piensa de una forma con respecto al aborto, pero el común de la gente por ahí piensa que es un acto de matar a alguien y no lo toma de la forma como uno, que ve las complicaciones de los abortos.

PV: En los profesionales, el valor de la complicación asociada a la muerte materna tiene un peso muy importante en lo que digan. Habría que hacer esta misma encuesta en una población grande que no tiene mucho conocimiento 
de esto. La gente no sabe muy bien". (Servicio de Obstetricia, Hospital S, grupo de médicos de planta)

PV: Quizás nosotros lo veamos desde el lado que vemos morir pacientes por aborto provocado. Y, bueno, desde ese lado uno piensa que es necesario un debate para ver cómo se pueden prevenir estas muertes. El que es religioso o la mayoría de la población no están en contacto con estas realidades. (Servicio de Ginecología, Hospital $\mathrm{M}$, grupo de residentes)

Esta visión se basa en el desconocimiento que los médicos tienen del estado de la opinión pública. Las encuestas indican que la población piensa de manera muy similar a los médicos/as respecto de cuáles son las situaciones en las que el aborto no debería estar penalizado: peligro de la vida de la mujer, embarazo producto de violación y feto con malformación incompatible con la vida extrauterina. ${ }^{13,14}$

\section{Discusión}

Los resultados presentados permiten concluir que:

1. El aborto y la anticoncepción son problemas que los médicos/as consideran muy relevantes para la salud pública.

2. La asignación de prioridades en las acciones parece tomar en cuenta la factibilidad de su implementación. Las propuestas son pragmáticas; se centran en aquello que se "puede" hacer en el corto plazo y sin requerir cambios importantes. Cabe pensar que los entrevistados/as tienen presente los avances y cambios ligados a la anticoncepción, como también el estancamiento de la situación normativa respecto del aborto.

3. La relación entre anticoncepción y aborto es vista como una relación causa-efecto (las fallas en el acceso a la planificación familiar constituyen el principal determinante del aborto inducido) que legitima su intervención. La anticoncepción es el "tratamiento" que permite evitar la "desviación" (el aborto).

4. Aunque la preocupación por el aborto es clara y explícita, las soluciones que imaginan son más bien limitadas. Estos límites están definidos por su formación profesional, la visión que tienen de sí mismos como "empresarios morales"15 y las características del contexto. El acuerdo con la no penalización es mayor en aquellas situaciones en que los entrevistados tienen o pueden llegar a tener incumbencia profesional (riesgo de vida o salud de la mujer, violación o malformación incompatible con la vida extrauterina). No se registran acuerdos importantes con situaciones que podrían ser motivo de preocupación desde una visión integral de la salud de la mujer ni se considera la interrupción del embarazo como una prerrogativa de la mujer.

5. Las diferencias significativas en las opiniones de varones y mujeres respecto de la despenalización del aborto, abonan la discusión sobre las posibles implicancias de la feminización de la ginecología y la obstetricia sobre la práctica médica. Algunos estudios afirman que las médicas pueden estar más motivadas a reducir la tradicional fragmentación de la atención médica de las mujeres o que se comunican más plenamente con sus pacientes. ${ }^{16}$ Otros sostienen que aún cuando las médicas pueden tener mayor empatía con las pacientes, su estilo comunicacional y las indicaciones terapéuticas son semejantes a los de sus colegas varones debido a la similitud en su entrenamiento profesional. ${ }^{17}$

El rol central que los tocoginecólogos juegan en la atención y prevención de la salud reproductiva conlleva un grado de responsabilidad social que va más allá de su práctica asistencial y que tradicionalmente (por razones históricas, políticas y culturales que no resulta sencillo explicar aquí) ha estado invisibilizado en nuestro medio. La comunidad médica suele argumentar que su función social se acota al ámbito de la consulta, que se restringe a sus prácticas (manejo de recursos y procedimientos preventivos, diagnósticos y terapéuticos) y a los mensajes que comunican a los usuarios/as de los servicios de salud. De esta forma se desdibuja la dimensión público-política de su actividad profesional: su función de mediadores entre las decisiones de sus pacientes sobre cuestiones reproductivas y las políticas de salud, población y familia del estado y su papel en la instrumentación de las normas jurídicas. No obstante, las opiniones recogidas nos devuelven la imagen de una comunidad profesional que, habiéndose formado y desempeñado buena parte de su práctica profesional en un contexto legal y cultural muy restrictivo, tiene una cierta sensibilidad respecto de las cuestiones más críticas del campo de la salud reproductiva y reconoce el papel del estado como garante de los derechos reproductivos y sexuales.

Con base en estos hallazgos, esbozamos ahora algunas líneas para el trabajo de abogacía con esta comunidad profesional. En primer lugar, podría trabajarse en la promoción de una visión más integral de la salud reproductiva y en la difusión de las perspectivas de género y derechos tanto a nivel de la formación profesional como de la actualización permanente. En segundo lugar, dar a conocer a los médicos/as por medio de las sociedades científicas un conjunto de documentos y evidencias. Entre otros: a) los pronun- 
ciamientos de las sociedades científicas internacionales respecto de la responsabilidad publico-política de los tocoginecólogos 2 ; b) las experiencias que -con la activa participación de sus pares- se están desarrollando en otros países de la región (por ejemplo, la realización de los abortos no punibles en hospitales públicos en Brasil o la difusión de la anticoncepción de emergencia en Uruguay) y c) la opinión de la ciudadanía respecto de los temas considerados "conflictivos" (educación sexual, despenalización del aborto, etc.). Finalmente, dado el fuerte peso que aún tiene en el discurso de los profesionales entrevistados el temor a la sanción moral y/o legal, resulta prioritario crear -sea mediante normas en los servicios, sea mediante la flexibilización de la legislación vigente- mejores condiciones para que los y las médicas puedan cumplir cabalmente con su responsabilidad profesional.

\section{Agradecimientos}

Este estudio recibió apoyo financiero del Programa Especial de Investigación, Desarrollo y Capacitación en Reproducción Humana de la Organización Mundial de Salud y de la Secretaría de Ciencia y Técnica del Ministerio de Educación y Cultura de la Nación.

Agradecemos los comentarios de John Townsend y Armando Seuc durante el Taller de Publicación y Difusión de Resultados, organizado por la Organización Mundial de la Salud y el Instituto Nacional de Salud Pública (Cuernavaca, Morelos, México, mayo de 2003). También agradecemos los aportes y la colaboración de Armando Seuc en el procesamiento de los datos y los comentarios de Kate Kostrzewa.

\section{Referencias}

I. Conrad P, Schneider J. Deviance and medicalization. From badness to sickness. Columbus: Merril Publishing Company, 1985.

2. Federación Internacional de Ginecología y Obstetricia (FIGO) y Organización Mundial de la Salud (OMS). Aborto: una responsabilidad profesional de los obstetras y los ginecólogos, Relatorio final. Grupo de trabajo FIGO/OMS. CEMICAMP, Campinas, San Pablo, Brasil, 2-5 de marzo de 1997.
3. Faúndes $A$, Leocádio $E$,Andalaft J. Making legal abortion accesible in Brazil. Reprod Health Matters 2002; I0(19):I20-I27.

4. McNaughton HL, Blandón MM,Altamirano L. Should therapeutic abortion be legal in Nicaragua:The response of Nicaraguan obstetriciangynaecologists. Reprod Health Matters 2002; I0(19): I I I-I I9.

5. Ramos S, Gogna M, Petracci M, Romero M, Szulik D. Los médicos frente a la anticoncepción y el aborto: ¿una transición ideológica? Buenos Aires: CEDES, 2001.

6. Área Salud, Economía y Sociedad del CEDES. La salud y los derechos sexuales y reproductivos: 2000-2003, un período de avances y retrocesos. En: CELS. Derechos Humanos en la Argentina. Informe 2002-2003. Buenos Aires: CELS, 2003:347-376.

7. Gutiérrez MA, Gogna M, Ramos S. Hacia nuevas formas de relación entre la sociedad civil y el Estado: la experiencia de Mujeres Autoconvocadas para Decidir en Libertad (Argentina). En: Dória Bilac E, Baltar da Rocha MI, comp. Saúde Reprodutiva na América Latina e no Caribe. Temas e Problemas. Campinas: PROLAP,ABEP, NEPO/UNICAMP/ Sao Paulo, 1998: 183-208.

8. FASGO (Federación Argentina de Sociedades de Obstetricia y Ginecología),AAGOP (Asociación Argentina de Ginecología y Obstetricia Psicosomática),ASAPER (Asociación Argentina de Perinatología),AMADA (Asociación Médica Argentina de Anticoncepción), SAP (Sociedad Argentina de Pediatría),ASAIGO (Asociación Argentina para el Estudio de las Infecciones en Ginecología y Obstetricia) et al. Solicitada en apoyo del Programa Nacional de Salud Sexual y Procreación Responsable. Buenos Aires: Diarios Clarín, La Nación y Diario Página 122003 junio 20:12.

9. Motta C, Rodríguez M. Mujer y justicia: el caso argentino. Buenos Aires: Banco Mundial, 2001:31.

10. Petracci M. Salud, derechos y opinión pública. Buenos Aires: Norma, 2004.

I I.Academia Nacional de Medicina. Solicitada. Buenos Aires: Diarios Clarín y La Nación, 1994 Agosto 4.

12. Rance S, Parras M. Aborto e anticoncepção na interação da consulta médica. Um estudo de caso: En: de Olivera Costa A, org. Direitos tardios: saúde, sexual e reprodução na América Latina. São Paulo: Fundação Carlos Chagas, Editora 34:225-248.

13. Muraro H. Nota sobre el estado de la opinión pública metropolitana acerca del debate sobre prohibición del aborto. Informe final de investigación presentado al Consejo Nacional de la Mujer, Buenos Aires: Norma, 2004.

14. Lista C. El debate sobre la despenalización del aborto:Androcentrismo y consenso informado. En: CENEP/OMS-CEDES-AEPA. Segundo Taller de Investigaciones Sociales en Salud Reproductiva y Sexualidad; 1996 mayo 6-7; Buenos Aires: 227-238.

I5. Freidson E. La profesión médica. Un estudio de sociología de conocimiento. Barcelona: Península, 1978.

16. Kutner N, Brogan D. Gender Roles, Medical Practice Roles, and Ob-Gyn Career Choice:A Longitudinal Study.Women \& Health 1990;16:3-4.

17. Mattila-Lindy S, Hemminki E, Malin M, Makkonen K, Topo P, Mantyranta T, et al. Physician's Gender and Clinical Opinions of Reproductive Health Matters. Women Health 1997;26(3):15-26. 